\title{
Form factors of pions, kaons \& protons at the highest timelike momentum transfers \& first measurements of hyperons
}

\author{
Kamal K. Seth ${ }^{1 a}$ \\ ${ }^{1}$ Northwestern University, Evanston, IL 60208, USA
}

\begin{abstract}
Electromagnetic form factors of hadrons for timelike momentum transfers can be measured by particle-antiparticle annihilation into hadron-antihadron pairs. We have made the world's first high precision measurements of timelike form factors of $\pi$ and $\mathrm{K}$ mesons, and ground states of the baryons, protons, and the hyperons $\Lambda^{0}, \Sigma^{0}, \Sigma^{+}, \Xi^{0}, \Xi^{-}$and $\Omega^{-}$at the high momentum transfers of 14.2 and $17.4 \mathrm{GeV}^{2}$. Limitations of perturbative QCD are revealed, and evidence is presented for the effects of diquark correlations in $\Lambda^{0}$ and $\Sigma^{0}$.
\end{abstract}

\section{Preamble}

An elementary particle is defined, or should be defined, as one which is not composite. So, all we want to know about an elementary particle is its static properties, mass, charge, spin, etc. When, almost exactly 100 years ago, Rutherford discovered the nucleus, we had just two elementary particles, the electron, and the proton, and there was no question of their constituents. While the electron has remained elementary, we know today that nucleons, proton and neutron, are not. The nucleons are composite of three light, spin $1 / 2(\mathrm{~m}<10 \mathrm{MeV}$ each, charge $+2 / 3$ and $-1 / 3)$ up and down quarks, which are bound by massless spin 1 gluons. Mesons are simpler, being composite of a quark and an antiquark, with the quarks being up, down, and strange.

Hyperons are baryons like the nucleons, with one or more up/down quarks replaced by strange quarks, which are still rather light. Now that mesons and baryons are composite, we have to find answers to a lot of questions. Among them, the important ones are:

a) How does one account for the masses of light quark mesons and baryons, their spins and their spatial and momentum distributions?

b) How do kaons differ from pions, and hyperons differ from nucleons?

c) What happens to the structure of a hadron when lots of momentum or energy is pumped into it from the outside?

The questions are of fundamental importance, and they can only be answered by experimental measurements. The measurements in question are form factor measurements.

We are all familiar with the fact that measurement of elastic scattering of electrons from nuclei and nucleons is related to spacelike electric and magnetic form factors, and they have provided direct insight into the spatial distribution of charges and currents in the nuclei, and in the nucleon. Unfortunately, this can only be done for protons and nuclei which are available as targets. Other

\footnotetext{
ae-mail: kseth@northwestern.edu
} 
baryons and mesons are not available as targets, and so spacelike form factors of strange quark baryons (hyperons), charm- or beauty-baryons, and mesons like pions and kaons are not measurable by elastic scattering. Fortunately, equally important complementary measurements, measurements of timelike form factors can be made for any hadron, and that is what I am going to talk about.

Since mesons contain only one quark and one antiquark, meson form factors provide simpler and more direct insight into the questions posed for baryons. So, I will first talk about meson form factors.

\section{Preliminaries}

Four momentum transfer, defined as

$$
\mathrm{Q}(4 \text { mom. })^{2}=\mathrm{q}(3 \mathrm{mom} .)_{\text {space }}^{2}-(\text { energy })_{\text {time }}^{2}
$$

can be positive and spacelike, or negative and timelike, as shown in Figure 1.
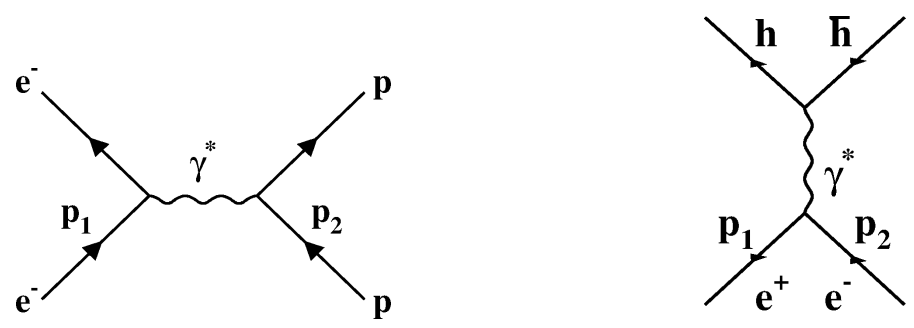

Figure 1. Left: spacelike form factor (elastic scattering); Right: timelike form factor (annihilation).

Form factors are analytic functions of momentum transfer, and therefore, a la Cauchy, for infinite momentum transfer

$$
\text { FF }\left(\text { spacelike, } Q^{2}=\infty\right)=\mathrm{FF}\left(\text { timelike, } Q^{2}=\infty\right)
$$

Because protons are available as targets, most of the early measurements were of spacelike form factors of protons via electron elastic scattering, $e+p \rightarrow e^{\prime}+p$. In 1960, the first proposals for electron-positron colliders were being considered at SLAC and Frascati. In anticipation of these, Cabibbo and Gatto wrote two classic papers $[1,2]$ pointing out that these colliders would provide the unique opportunity to measure timelike form factors of any hadrons, mesons and baryons by $e^{+} e^{-} \rightarrow$ $h \bar{h}$.

For baryons, there are two form factors, the Pauli and Dirac form factors, or more familiarly, the magnetic $G_{M}(s)$ and the electric $G_{E}(s)$ form factors. For $e^{+} e^{-} \rightarrow p \bar{p}$ the differential cross section is

$$
\frac{d \sigma_{0}(s, \theta)_{B}}{d \Omega}=\frac{\alpha^{2}}{4 s} \beta_{B}\left[\left|G_{M}^{B}(s)\right|^{2}\left(1+\cos ^{2} \theta\right)+\tau / 2\left|G_{E}^{B}(s)\right|^{2} \sin ^{2} \theta\right]
$$

At large squared momentum transfers, $\left|Q^{2}\right| \equiv s$, the quantity $\tau=4 m_{p}^{2} / s$ becomes small, the contribution of $G_{E}^{B}$ becomes small, and it becomes difficult to determine it. According to the dimensional counting rule of QCD [3], $G_{M}^{B}(s)$ vary as s ${ }^{-2}$, and the above cross section decreases as s ${ }^{-5}$, making it extremely difficult, if not impossible, to measure baryon form factors for $\left|Q^{2}\right|>20 \mathrm{GeV}^{2}$.

For pseudoscalar mesons, $\pi$ and $\mathrm{K}$, with zero spin, there is only one form factor, $F_{m}(s)$, and the differential cross section is

$$
\frac{d \sigma_{0}(s, \theta)_{m}}{d \Omega}=\frac{\alpha^{2}}{4 s} \beta_{m}\left|F_{m}(s)\right|^{2} \sin ^{2} \theta
$$

Further, dimensional counting rules predicts that $F_{m}(s)$ varies as $\mathrm{s}^{-1}$ [3], and the cross sections decrease only as $\mathrm{s}^{-3}$, making life at large $\left|\mathrm{Q}^{2}\right|$ easier! 


\section{Prejudices \& Obstacles}

Two obstacles for measuring timelike form factors exist at a collider like CESR at Cornell. The first is the prejudice that only weak interaction flavor physics is important, the rest has little priority. It is an uphill battle to get the required beam time allocated for other measurements, and certainly timelike form factors are not weak interaction physics. The second obstacle is more generic. Everybody loves resonances, and they love to run measurements on peaks of resonances. So, most of the data taken at CLEO-c was taken at the peaks of $\psi(n S)$ resonances. To use these data for form factor measurements one has to show that the strong decay of these resonances, $e^{+} e^{-} \rightarrow \psi(n S) \rightarrow$ 3gluons $\rightarrow h \bar{h}$ have negligibly small cross sections for decay into the hadron pairs of our interest, i.e., $\sigma(R) \rightarrow h \bar{h}$. For the bound vector states of charmonium, $J / \psi$ and $\psi(2 S)$, strong interaction decays are dominant. However, the resonances above the $D \bar{D}$ threshold, $\psi(3 S, 3772)$ and $\psi(4 S, 4160)$ mainly decay to $D \bar{D}$, and the branching fractions for their decay to $h \bar{h}$ are expected to be very small. The resonance cross sections can be estimated using the pQCD relation [4] that

$$
\mathcal{B}\left(\psi\left(n^{\prime}\right)\right) / \mathcal{B}(\psi(n)) \text { to hadrons }=\mathcal{B}\left(\psi\left(n^{\prime}\right)\right) / \mathcal{B}(\psi(n)) \text { to leptons }
$$

Since, $\mathcal{B}\left(\psi(3770), \psi(4160) \rightarrow e^{+} e^{-}\right) / \mathcal{B}\left(\psi(3686) \rightarrow e^{+} e^{-}\right)=(0.36,1.04) \times 10^{-3}$, it follows that the branching fractions for the hadronic decays of $\psi(3770)$ and $\psi(4160)$ are more than three orders of magnitude smaller than the corresponding measured decays of $\psi(3686)$.

With nearly 5 million $\psi(3770)$ and $\psi(4160)$ each, formed in the present measurements, and our detection efficiencies, we estimate the number of resonance events for the decays as follows:

$\begin{array}{ccccccccc}\boldsymbol{\pi}^{+} \boldsymbol{\pi}^{-} & \boldsymbol{K}^{+} \boldsymbol{K}^{-} & \boldsymbol{p} \overline{\boldsymbol{p}} & \boldsymbol{\Lambda} \overline{\boldsymbol{\Lambda}} & \boldsymbol{\Sigma}^{+} \overline{\boldsymbol{\Sigma}}^{+} & \boldsymbol{\Sigma}^{\mathbf{0}} \overline{\boldsymbol{\Sigma}}^{\mathbf{0}} & \boldsymbol{\Xi}^{-} \overline{\boldsymbol{\Xi}}^{-} & \boldsymbol{\Xi}^{\mathbf{0}} \overline{\boldsymbol{\Xi}}^{\mathbf{0}} & \boldsymbol{\Omega}^{-} \overline{\boldsymbol{\Omega}}^{-} \\ \sim 0.04 & 0.4 & 1.3 & 0.9 & 0.2 & 0.2 & 0.2 & 0.05 & 0.03\end{array}$

The observed counts for each decay turn out to be about 100 times larger than these resonance contributions. Therefore, all observed $e^{+} e^{-} \rightarrow \pi \pi, K K, p \bar{p}$, and hyperon yields we observe can be safely attributed to form factors.

\section{Form Factors of Pions and Kaons}

Before 1990, almost no experimental data with any precision existed for pion and kaon spacelike or timelike form factors for $\left|\mathrm{Q}^{2}\right|>5 \mathrm{GeV}^{2}$. Historical note: Recall the famous Brodsky versus Isgur/Llwellyn-Smith debate (1984-1989) on when the momentum transfer is large enough for perturbative QCD to be valid. At that time, the discussion could only use the small amount of small $\mathrm{Q}^{2}$ data for $\mathrm{F}_{\pi}$ with larger errors which was available then. The picture is different now.

Spacelike form factors of mesons are very difficult to measure, because meson targets do not exist. Two different methods have been used.

1) $F_{\pi}$ and $F_{K}$ from Elastic Scattering of pions/kaons off atomic electrons, $\pi(K) e^{-} \rightarrow \pi(K) e^{-}$. Unfortunately, in this approach the momentum transfer is very small. At CERN for $200 \mathrm{GeV}$ pions, $\mathrm{Q}^{2}(\pi) \leq 0.25 \mathrm{GeV}^{2}$ and $\mathrm{Q}^{2}(\mathrm{~K}) \leq 0.11 \mathrm{GeV}^{2}$ were realized.

2) $\mathrm{F}_{\pi}$ from electroproduction of pions, $\mathrm{e}^{-} \mathrm{p} \rightarrow \mathrm{e}^{-} \pi^{+} \mathrm{n}$, has serious theoretical problems and uncertainties. The good precision data are confined to $\mathrm{Q}^{2}<2.45 \mathrm{GeV}^{2}$ [5]. For kaons no electroproduction data exist, and no spacelike form factors have been measured.

In the following we present our excellent precision timelike form factor results for $\pi$ and $\mathrm{K}$ at large $\mathrm{Q}^{2}=14.2$ and $17.4 \mathrm{GeV}^{2}[6]$. The data were taken with the CLEO-c detector for $e^{+} e^{-}$annihilation at $\sqrt{s}=3.772 \mathrm{GeV}$ and $4.170 \mathrm{GeV}$ with luminosities of 805 and $586 \mathrm{pb}^{-1}$, respectively. 


\section{The CLEO-c Detector}

The CLEO-c detector is a cylindrical general purpose detector, as shown in Figure 2. The detector components important for the present measurements are the CsI electromagnetic calorimeter, the drift chamber for charged particle detection, and the RICH detector, all of which are located in a 1 Tesla solenoidal magnetic field. The acceptance for photons and charged particles in the central detector is $|\cos \theta|<0.8$.

Charged particle resolution is $\sigma_{p} / p=0.6 \% @ 1 \mathrm{GeV} / \mathrm{c}$. Photon resolution is $\sigma_{E} / E=2.2 \% @ 1$ $\mathrm{GeV}$, and $5 \% @ 100 \mathrm{MeV}$. The data we use consists of $805 \mathrm{pb}^{-1}$ at $\psi(3770),\left|\mathrm{Q}^{2}\right|=14.2 \mathrm{GeV}^{2}$, and $586 \mathrm{pb}^{-1}$ at $\psi(4170), \mathrm{QQ}^{2} \mathrm{I}=17.4 \mathrm{GeV}^{2}$.

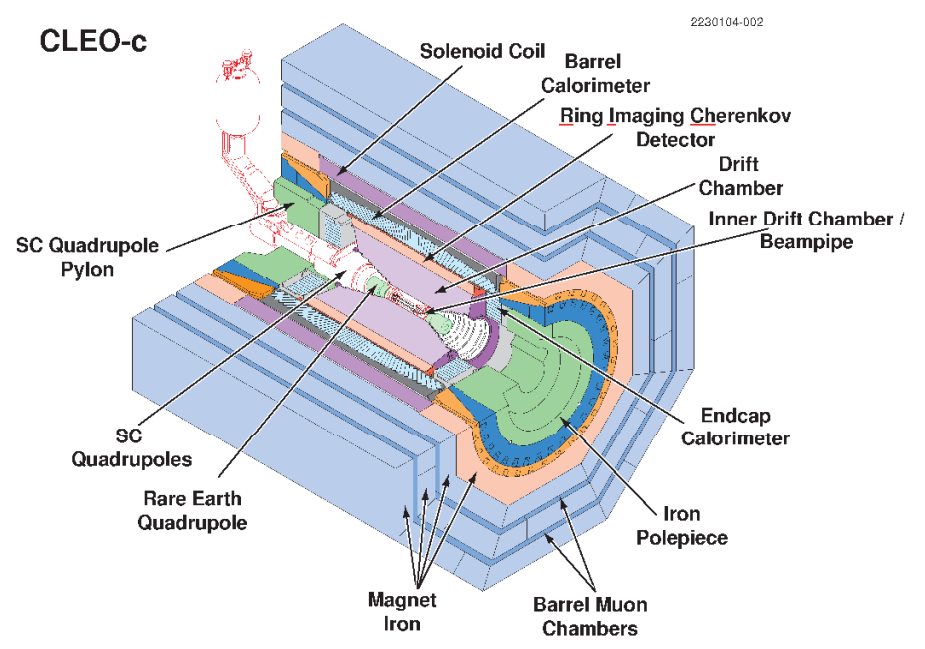

Figure 2. A schematic view of the CLEO-c detector.

Timelike form factors of any hadron can be determined by measuring $\sigma\left(e^{+} e^{-} \rightarrow h \bar{h}\right)$, but one has to reject 3 to 4 orders of magnitude larger background of QED-produced $\mathrm{e}^{+} \mathrm{e}^{-}$and $\mu^{+} \mu^{-}$pairs, and substantial tails of lighter hadrons. This is illustrated by Monte Carlo simulations shown in Figure 3.

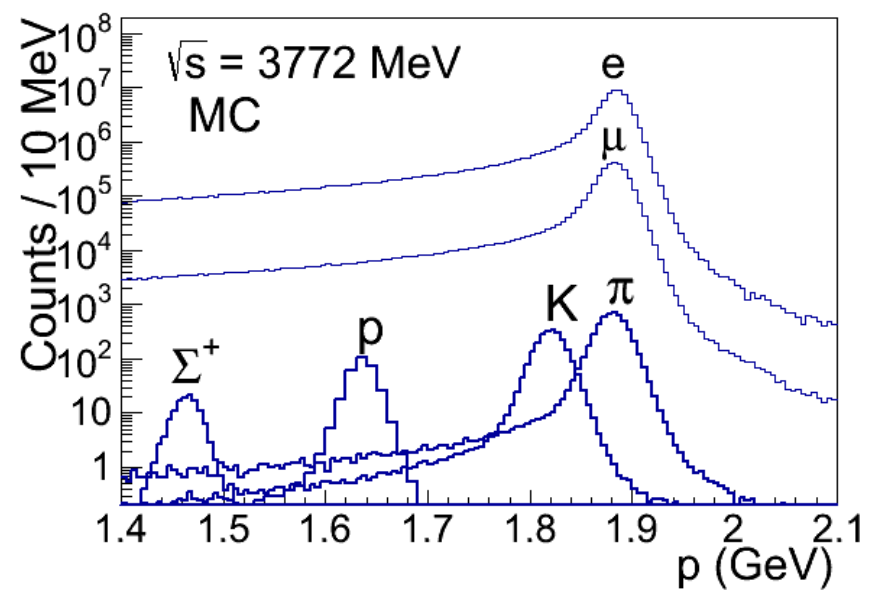

Figure 3. Monte Carlo simulation of momentum distributions expected for $e^{+} e^{-}(\sqrt{s}=3.772 \mathrm{GeV}) \rightarrow$ $e^{+} e^{-}, \mu^{+} \mu^{-}$, and $h \bar{h}$. Notice that $e^{+} e^{-}$and $\mu^{+} \mu^{-}$yields are $10^{3}$ to $10^{4}$ times larger than all hadronic yields. 

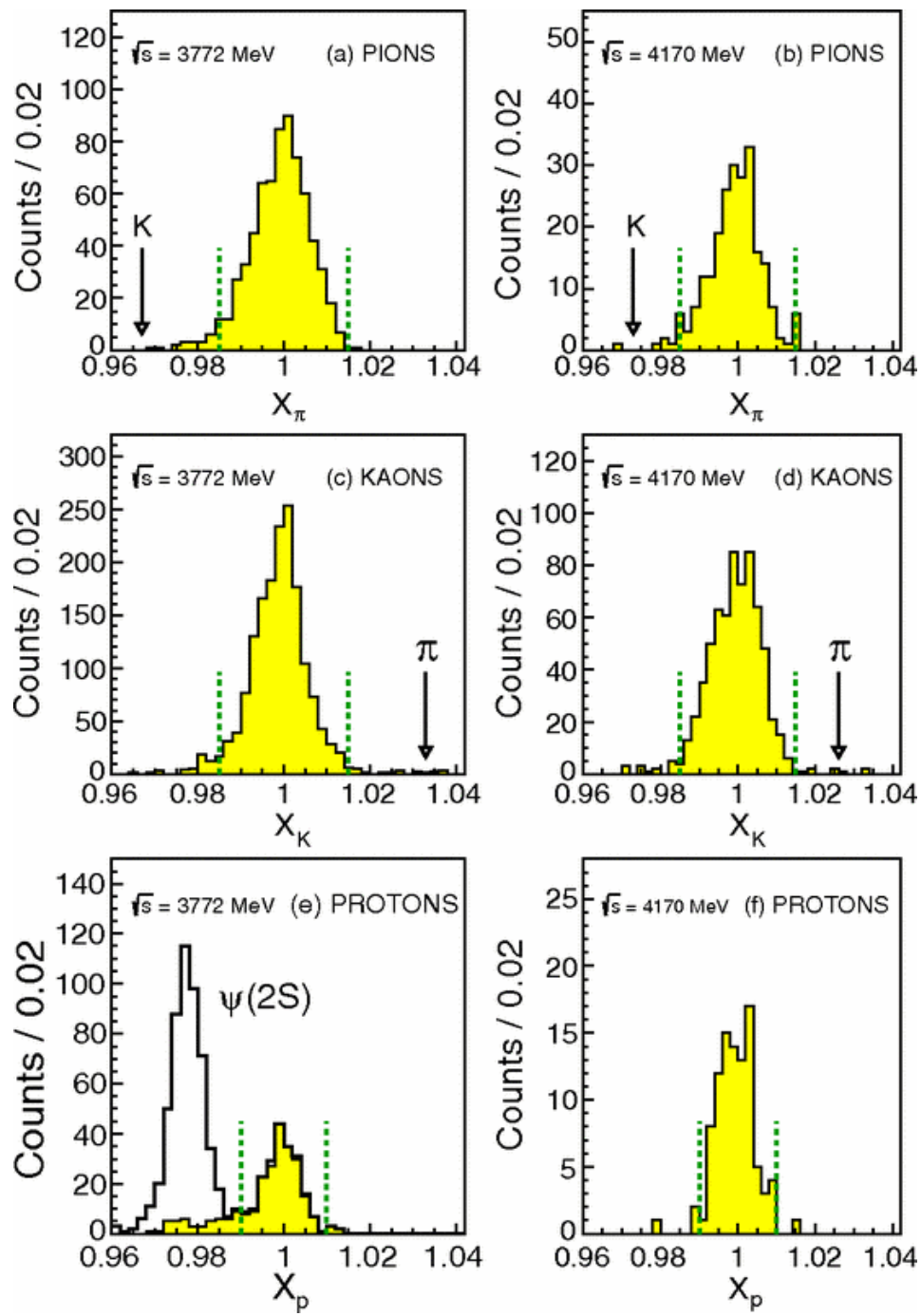

Figure 4. Distributions of $X_{h} \equiv[E(h)+E(\bar{h})] / \sqrt{s}$ for data at $\sqrt{s}=3.77$ and $4.17 \mathrm{GeV}$.

I will not bore you with the nitty-gritty of particle identification, but Figure 4 illustrates, in terms of the observable $X_{h} \equiv[E(h)+E(\bar{h})] / \sqrt{s}$, that using the excellent capabilities of the CLEO-c detector we were able to reject electron and muon contaminations essentially perfectly, and obtain clean distributions of pions, muons, and protons with essentially no background.

Our results for pions, kaons and protons are presented in Figures 5-7. Figure 5 shows that the angular distributions for pions and kaons at both $\sqrt{s}=3772 \mathrm{MeV}$ and $4170 \mathrm{MeV}$ fit very well the $\sin ^{2} \theta$ distribution expected for electric form factors [7]. There is little evidence for $\left(1+\cos ^{2} \theta\right)$ distribution (dashed) contribution expected for a finite magnetic form factor which is possible in principle because of the spins of the quarks in the pseudoscalar pions and kaons.

Figure 6 and Table 1 show our form factor results. The important experimental results are:

1. There is a remarkable agreement of the form factors for both pions and kaons with the dimensional counting rule prediction of $Q C D$, that $\mathrm{QQ}^{2} \mid \mathrm{F}_{\pi, \mathrm{K}}$ are nearly constant, varying with $\mid \mathrm{Q}^{2} \mathrm{I}$ only weakly as $\alpha_{S}\left(\left|Q^{2}\right|\right)[3]$. 
2. The existing theoretical $\mathrm{pQCD}$ and $\mathrm{QCD}$ sum-rule predictions for pions underpredict the magnitude of $F_{\pi}\left(\left|Q^{2}\right|\right)$ at large $\left|Q^{2}\right|$ by large factors, $\geq 2$. There are no detailed predictions for kaons [8].

3. Perturbative QCD predicts that $\mathrm{F}_{\pi} / \mathrm{F}_{\mathrm{K}}=\left(\mathrm{f}_{\pi} / \mathrm{f}_{\mathrm{K}}\right)^{2}=0.67 \pm 0.01$. We find: $\mathrm{F}_{\pi} / \mathrm{F}_{\mathrm{K}}=1.21 \pm 0.03$, at $\left|Q^{2}\right|=14.2 \mathrm{GeV}^{2}, \mathrm{~F}_{\pi} / \mathrm{F}_{\mathrm{K}}=1.09 \pm 0.04$, at $\left|\mathrm{Q}^{2}\right|=17.4 \mathrm{GeV}^{2}$. This $>50 \%$ disagreement is extremely surprising, and needs to be understood.
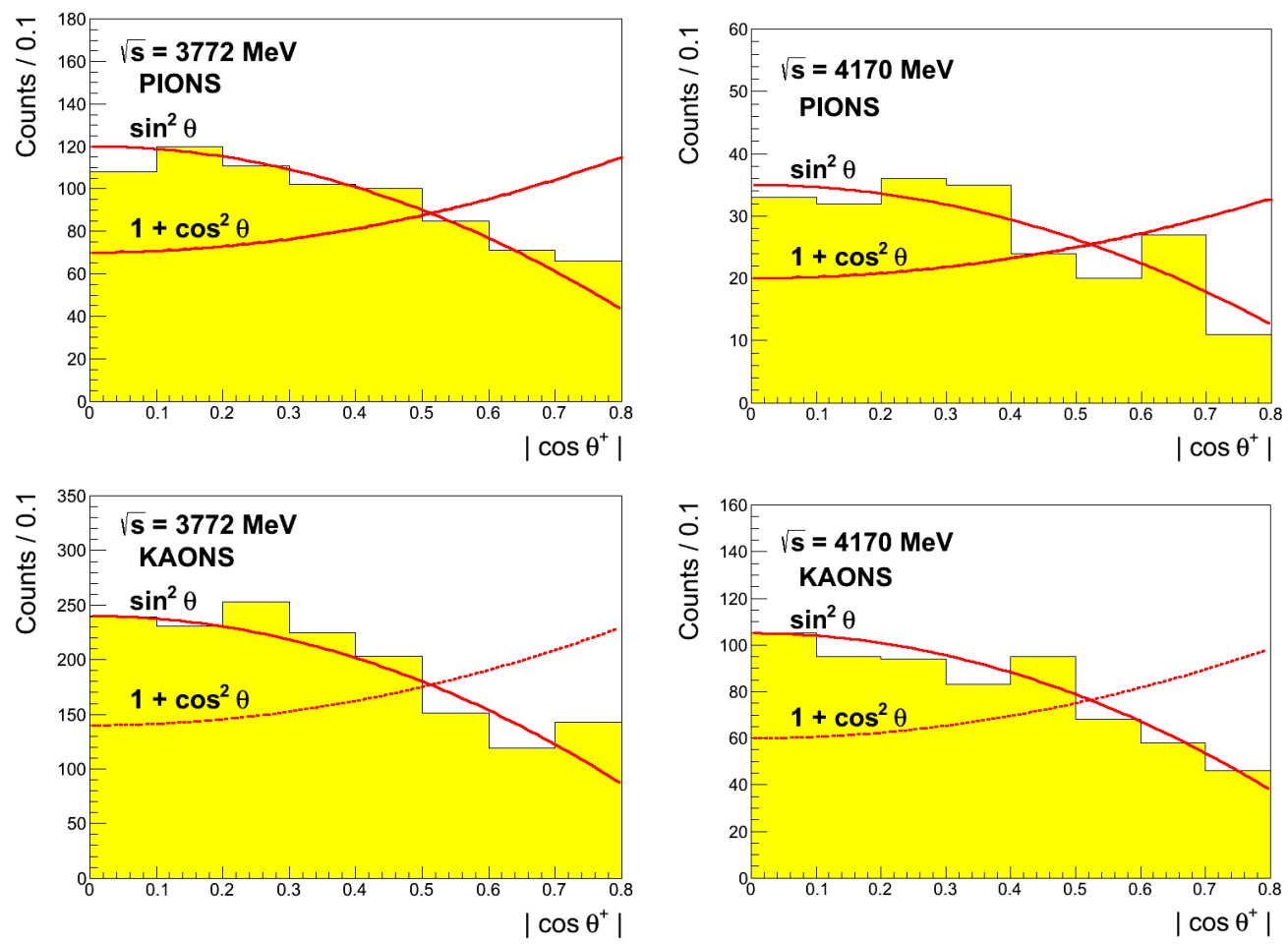

Figure 5. Angular distributions for pion and kaon form factor events.
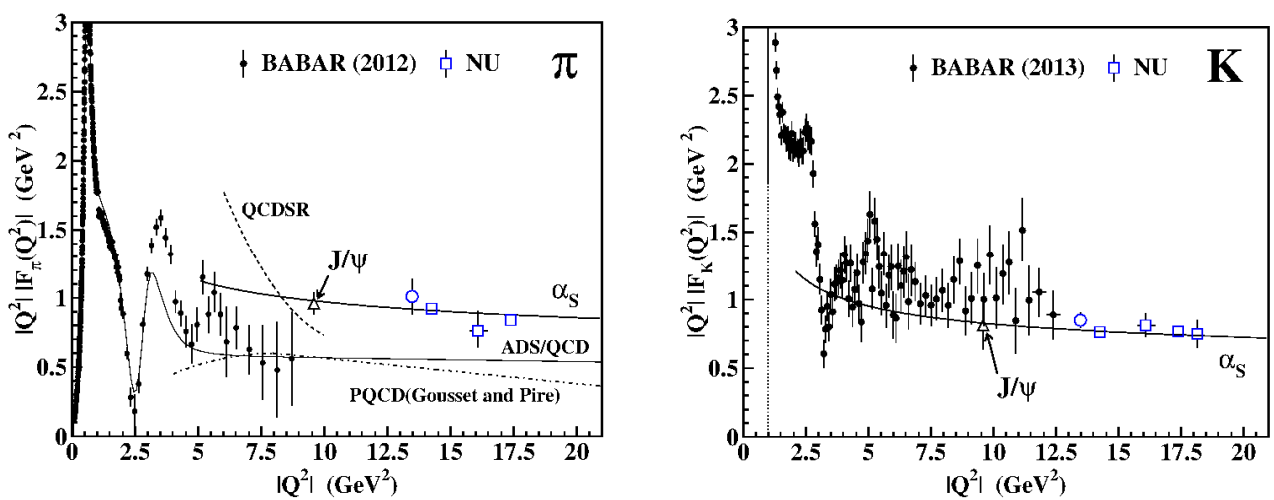

Figure 6. Timelike form factors for pions and kaons. Notice the excellent confirmation of the QCD dimensional counting rule prediction of $\left|\mathrm{Q}^{-2}\right|$ variation of $F_{\pi}$ and $F_{K}$ by the present results for $\left|\mathrm{Q}^{2}\right|>12.5 \mathrm{GeV}[6]$. 
Table 1. Timelike form factors for pions and kaons. Notice the excellent confirmation of the QCD dimensional count up rule prediction of $\left|\mathrm{Q}^{-2}\right|$ variation of $F_{\pi}$ and $F_{K}$ by the present results for $\left|\mathrm{Q}^{2}\right|>12.5 \mathrm{GeV}[6]$.

\begin{tabular}{ccccc}
\hline$Q^{2}\left(\mathrm{GeV}^{2}\right)$ & $\boldsymbol{N}\left(\boldsymbol{\pi}^{+} \boldsymbol{\pi}^{-}\right)$ & $\sigma($ Born $), \mathrm{pb}$. & $F_{\pi}\left(\left|Q^{2}\right|\right) \times 10$ & $Q^{2} F_{\pi}\left(\left|Q^{2}\right|\right)\left(\mathrm{GeV}^{2}\right)$ \\
\hline $\mathbf{1 3 . 5}$ & $\mathbf{2 6} \pm \mathbf{5}$ & $\mathbf{9 . 0} \pm \mathbf{1 . 8}$ & $\mathbf{0 . 7 5} \pm \mathbf{0 . 0 9}$ & $\mathbf{1 . 0 2} \pm \mathbf{0 . 1 3}$ \\
$\mathbf{1 4 . 2}$ & $\mathbf{6 6 1} \pm \mathbf{2 6}$ & $\mathbf{6 . 3 6} \pm \mathbf{0 . 2 5}$ & $\mathbf{0 . 6 5} \pm \mathbf{0 . 0 1}$ & $\mathbf{0 . 9 2} \pm \mathbf{0 . 0 4}$ \\
$\mathbf{1 7 . 4}$ & $\mathbf{2 1 3} \pm \mathbf{1 2}$ & $2.89 \pm \mathbf{0 . 1 6}$ & $\mathbf{0 . 4 8} \pm \mathbf{0 . 0 1}$ & $\mathbf{0 . 8 4} \pm \mathbf{0 . 0 3}$ \\
\hline$Q^{2}\left(\mathrm{GeV}^{2}\right)$ & $\boldsymbol{N}\left(\boldsymbol{K}^{+} \boldsymbol{K}^{-}\right)$ & $\sigma($ Born$), \mathrm{pb}$. & $F_{K}\left(\left|Q^{2}\right|\right) \times 10$ & $Q^{2} F_{K}\left(\left|Q^{2}\right|\right)\left(\mathrm{GeV}^{2}\right)$ \\
\hline $\mathbf{1 3 . 5}$ & $\mathbf{7 1} \pm \mathbf{9}$ & $\mathbf{5 . 7} \pm \mathbf{0 . 7}$ & $\mathbf{0 . 6 3} \pm \mathbf{0 . 0 4}$ & $\mathbf{0 . 9 1} \pm \mathbf{0 . 1 4}$ \\
$\mathbf{1 4 . 2}$ & $\mathbf{1 5 6 4} \pm 40$ & $\mathbf{3 . 9 5} \pm \mathbf{0 . 1 0}$ & $\mathbf{0 . 5 4} \pm \mathbf{0 . 0 1}$ & $\mathbf{0 . 7 6} \pm \mathbf{0 . 0 2}$ \\
$\mathbf{1 7 . 4}$ & $\mathbf{6 4 4} \pm \mathbf{2 5}$ & $\mathbf{2 . 2 3} \pm \mathbf{0 . 0 9}$ & $\mathbf{0 . 4 4} \pm \mathbf{0 . 0 1}$ & $\mathbf{0 . 7 7} \pm \mathbf{0 . 0 3}$ \\
\hline
\end{tabular}

\section{Theoretical Implications}

Lattice lives in Euclidean time, and is not capable of addressing timelike form factors. So we expect no lattice-based predictions for form factors, and have to live with predictions based on QCD-based models for timelike form factors. The starting point of the existing calculations is factorization, with $F\left(Q^{2}\right)=\psi_{\text {in }} \times T_{H} \times \psi_{\text {out }}$. The meson wave functions $\psi_{\text {in,out }}$ represent soft components, not calculable perturbatively. $\mathrm{T}_{\mathrm{H}}$ represents the hard interaction, "hopefully calculable in perturbative QCD." Since ab initio the quark wave functions are not known, various empirical wave functions have been used. Lepage and Brodsky used the asymptotic wave function $\phi(x)_{a s} \propto f_{\pi} x(1-x)$, where $q$ and $\bar{q}$ share momenta equally. Chernyak and Zhitnitsky used the QCD sum-rule-inspired wave function $\phi(x)_{C Z} \propto\left(1-2 x^{2}\right) \phi(x)_{a s}$ which produces a two humped distribution.

The pQCD expression [9] for form factors is $\left|Q^{2}\right| F_{\pi, K}\left(\left|Q^{2}\right|\right)=16 \pi \alpha_{S}\left|Q^{2}\right| \times f_{\pi, K}^{2}$.With the known decay constants $\mathrm{f}_{\pi}=130.41 \mathrm{MeV}$, and $\mathrm{f}_{\mathrm{K}}=156.1 \mathrm{MeV}$, this leads, for $\left|\mathrm{Q}^{2}\right|=17.4 \mathrm{GeV}^{2}$, to $\left|Q^{2}\right| F_{\pi}\left(\left|Q^{2}\right|\right)=0.21 \mathrm{GeV}^{2}$, and $\left|Q^{2}\right| F_{K}\left(\left|Q^{2}\right|\right)=0.31 \mathrm{GeV}^{2}$, factors 3 to 4 smaller than what we measure. This leads to the serious problem that even the ratio $F_{\pi} / F_{K}$, which is supposed to remove the dependence on the assumed identity of pion and kaon wave functions, and is predicted to be $F_{\pi} / F_{K}=f_{\pi}^{2} / f_{K}^{2}=0.67 \pm 0.01$, is $(39 \pm 4) \%$ smaller than our measurement of $1.09 \pm 0.04$ for $\left|\mathrm{Q}^{2}\right|=17.4 \mathrm{GeV}^{2}[6]$. With the precision of our measurements being at the level of $\sim 5 \%$, it is quite obvious that something is very wrong. Could it be the assumed identity of the $\pi$ and $\mathrm{K}$ wave functions? Could it be that pQCD is not valid even for $\left|Q^{2}\right|=17.4 \mathrm{GeV}^{2}$ ?

Since the relation $F_{\pi}\left(\left|Q^{2}\right|\right) / F_{K}\left(\left|Q^{2}\right|\right)=f_{\pi}^{2} / f_{K}^{2}$ is based on assuming identical wave functions for pions and kaons, Lepage and Brodsky [9] conjectured that because the s-quark in the kaon is $\sim 27$ times heavier than the $\langle u, d\rangle$ quarks in the pion, and the SU(3) flavor symmetry is broken, the kaon wave function may differ from the pion wave function by acquiring an asymmetric component, and this may account for the observed violation of the above relation. While large differences in pion and kaon wave functions were proposed by Chernyak \& Zhitnitsky, recent quenched lattice calculations [10], predict a much smaller asymmetric component in the kaon wave function, and a much smaller effect of SU(3)-breaking than CZ proposed. What is needed is an experimental measure of the effect of SU(3) breaking.

Lepage and Brodsky [9] predicted that a large SU(3) breaking effect would lead to a large form factor for the neutral kaon, and " $F_{K_{S} K_{L}} / F_{K^{+} K^{-}}$of the order one" at a large $\left|Q^{2}\right|$, and suggested that $F_{K_{S} K_{L}}$ should be measured. Following this suggestion, we have made the first ever measurement of the form factor $F_{K_{S} K_{L}}\left(\left|Q^{2}\right|\right)$ at $\left|\mathrm{Q}^{2}\right|=17.4 \mathrm{GeV}^{2}[11]$. To validate our event selection criteria, we also 
measure $\psi(2 S) \rightarrow K_{S} K_{L}$ for $\mid \mathrm{Q}^{2} \mathrm{I}=13.6 \mathrm{GeV}^{2}$, which has large yield. We use the same event selection criteria at $\left|Q^{2}\right|=17.4 \mathrm{GeV}^{2}$ to measure form factor events.

For $e^{+} e^{-} \rightarrow K_{S} K_{L}$ at $\sqrt{s}=4.17 \mathrm{GeV}$, we obtain 4 events in the signal region, and a Monte Carlo background estimate of 2 counts. This leads for $\left|Q^{2}\right|=17.4 \mathrm{GeV}^{2}$ to:

$$
F_{K_{S} K_{L}}\left(\left|Q^{2}\right|\right)=3.9 \times 10^{-3} \text {, and } F_{K_{S} K_{L}}\left(\left|Q^{2}\right|\right) / F_{K^{+} K^{-}}\left(\left|Q^{2}\right|\right)=0.09
$$

In other words, the SU(3) breaking effect on the ratio is found to be small, certainly much less than of "the order of one". To come back to the original problem of $F_{\pi} / F_{K}$ (expt.) $\neq f_{\pi}^{2} / f_{K}^{2}$, it is now apparent that it can not be attributed to SU(3) breaking alone. The problem remains unresolved.

Here is a challenge worthy of the best theoretical attempts.
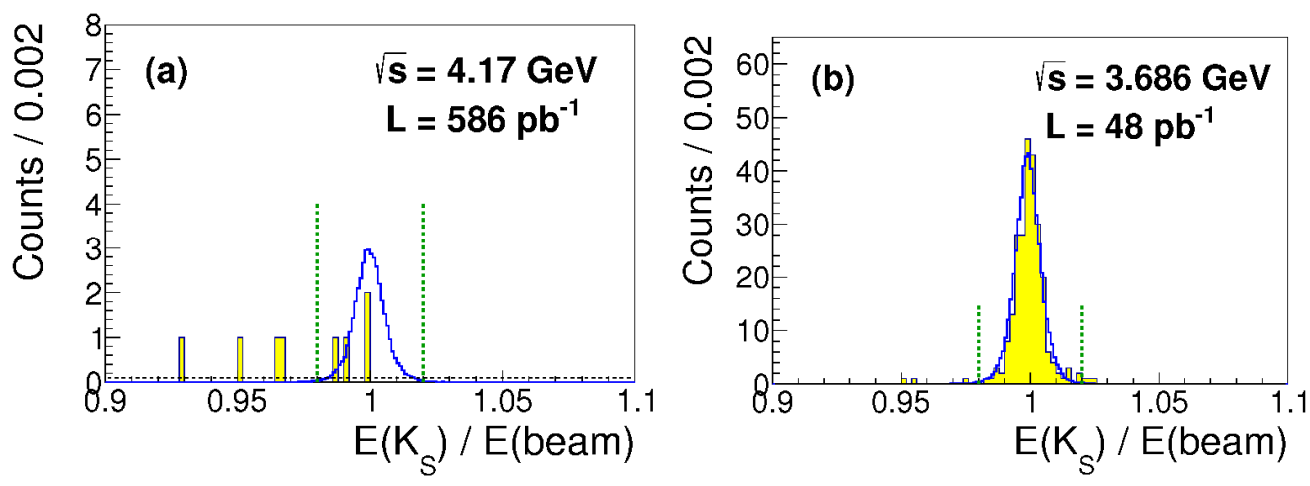

Figure 7. Measurements of $e^{+} e^{-} \rightarrow K_{S} K_{L}$. (a) $K_{S}$ form factor measurement at $\sqrt{s}=4.17 \mathrm{GeV}$, (b) $\psi(2 S)$ resonance measurement at $\sqrt{s}=3.686 \mathrm{GeV}$ [11].

\section{Timelike Form Factors of Protons}

Spacelike form factors of the proton have been measured since the 1980's, and precision measurements have existed for $\mathrm{Q}^{2}$ up to $31 \mathrm{GeV}^{2}$. Prior to 1993 , measurements of the timelike form factors of the proton by the reaction $e^{+} e^{-} \rightarrow p \bar{p}$ were sparse, had large errors, and were confined to $\left|\mathrm{Q}^{2}\right|<5.7 \mathrm{GeV}^{2}$. In 1993 , at Fermilab we measured $\mathrm{G}_{\mathrm{M}}\left(\left|\mathrm{Q}^{2}\right|\right)$ by $p \bar{p} \rightarrow e^{+} e^{-}$for $\left|\mathrm{Q}^{2}\right|=8.9$ to 13.11 $\mathrm{GeV}^{2}$ [12], as shown in Figure 8. While $\mathrm{Q}^{4} \mathrm{G}_{\mathrm{M}}\left(\left|\mathrm{Q}^{2}\right|\right)$ was found to vary as $\alpha^{2}$ (strong) above $9 \mathrm{GeV}^{2}$, as predicted by QCD counting rules, a big surprise was discovered. It was found that $G_{M}$ (timelike) $/ G_{M}$ (spacelike) $\approx 2$, in strong disagreement with the pQCD expectation of the two being equal at large momentum transfers.

Two possible explanations of the unexpected observation $G_{M}$ (timelike) $/ G_{M}($ spacelike $) \approx 2$, at $\left|Q^{2}\right|=8-13 \mathrm{GeV}^{2}$ were offered.

1) $\left|Q^{2}\right|=13 \mathrm{GeV}^{2}$ is not large enough for $\mathrm{pQCD}$ to be valid.

2) The quark distribution in the proton is not like a Mercedes star, with the three quarks having identical distributions, but like diquark-quark, with a preferential pairing of the two u-quarks [13].

$\mathbf{u}$

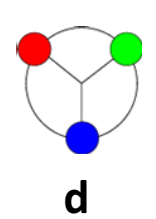

$\mathbf{u}$

$\mathbf{u}$

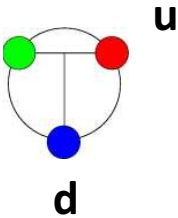


Although no alternate explanations have been offered, the diquark-quark model did not acquire acceptance despite the fact that it was successful [13] in explaining the $G_{M}(p)$ (timelike) $/ G_{M}(p)($ spacelike $) \approx 2$.

To test the validity of $\mathrm{pQCD}$ at larger $\mid \mathrm{Q}^{2} \mathrm{I}$, we have made high precision measurements of $G_{M}(p)$ for timelike $\left|Q^{2}\right|=14.2$ and $17.4 \mathrm{GeV}^{2}$, using data taken at the $e^{+} e^{-}$CESR collider at Cornell, and the detector CLEO-c. The results are shown in the right hand panel of Figure 8 . It is seen that $G_{M}(p)$ (timelike) $/ G_{M}(p)$ (spacelike) $\approx 2$ persists even for $\left|Q^{2}\right|$ up to $\approx 18 \mathrm{GeV}^{2}$. This suggests that the diquark model should be taken seriously. As we show later, the form factors of $\Lambda^{0}$ and $\Sigma^{0}$ hyperons offer new evidence for diquark correlations.
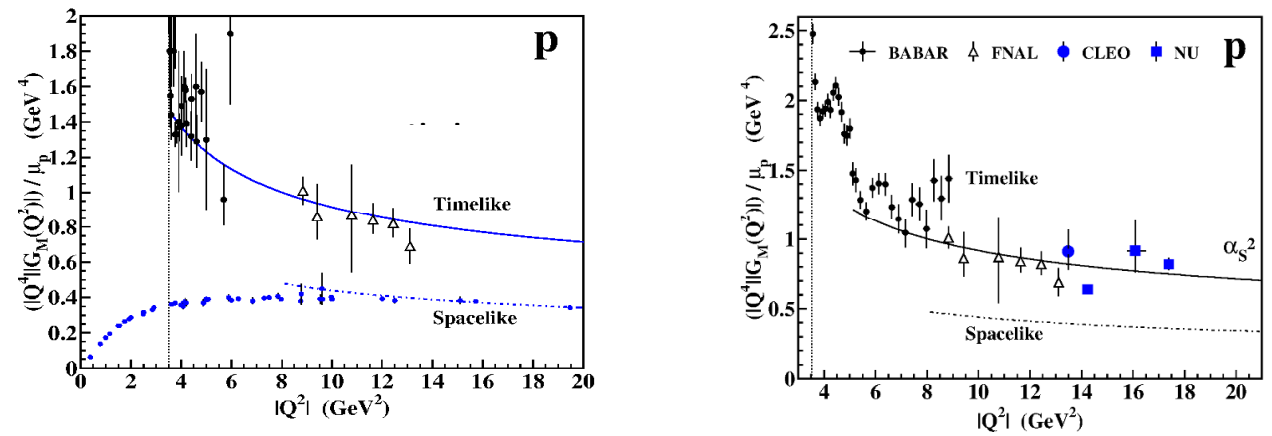

Figure 8. $\left|Q^{4}\right| G_{M} / \mu_{p}$ for timelike form factors of the proton as function of $\left|Q^{2}\right|$. Left panel: Pre-1993 $e^{+} e^{-} \rightarrow$ $p \bar{p}$ results with large errors are shown for $\left|Q^{2}\right|<5.7 \mathrm{GeV}^{2}$. Fermilab results for $p \bar{p} \rightarrow e^{+} e^{-}$in the region 8-13 $\mathrm{GeV}^{2}[12]$ show that timelike form factors are twice as large as the corresponding spacelike form factors [12]. Right panel: Including our new results in the $\left|Q^{2}\right|=14-18 \mathrm{GeV}^{2}$ region [6]. The timelike form factors remain factor two larger.

\section{Timelike Form Factors of Hyperons}

In 1960, before quarks were even proposed, but strangeness and strange baryons, the hyperons (as we call them now), were known, Cabibbo and Gatto wrote two classic papers on the measurement of timelike form factors by $\mathrm{e}^{+} \mathrm{e}^{-} \rightarrow$ hadron-antihadron [1,2]. They discussed the proton and neutron, and pion and kaon, and went on to say that it would be very interesting to measure hyperon form factors. But they noted that the cross sections are likely to be very small, and despaired whether they could be measured.

Fifty five years later we have now measured hyperon form factors for the first time with good precision at the large momentum transfer of $\left|Q^{2}\right|=14.2 \mathrm{GeV}^{2}[14]$. We identify the hyperons by their dominant decays. These (and their branching fractions) are: $\Lambda^{0} \rightarrow p \pi^{-}(64 \%), \Sigma^{+} \rightarrow p \pi^{0}$ (52\%), $\Sigma^{0} \rightarrow \Lambda \gamma(100 \%), \Xi^{-} \rightarrow \Lambda \pi^{-}(100 \%), \Xi^{0} \rightarrow \Lambda \pi^{0}(100 \%)$, and $\Omega^{-} \rightarrow \Lambda K^{-}(68 \%)$.

The study of hyperons allows us to study interesting questions. As we go from protons to hyperons, serially replacing one (in $\Lambda$ and $\Sigma$ ), two (in $\Xi$ ), or three (in $\Omega^{-}$) up/down quarks with strange quarks, what do we expect to learn at $\left|Q^{2}\right|=14.2 \mathrm{GeV}^{2}$ ? Do we see SU(3) breaking effects? Do we see diquark correlation effects? Are $G_{M}(B)$ for hyperons proportional to $\mu_{B}$, as for nucleons? Do neutral hyperons have finite $G_{E}\left(Q^{2}\right)$ as the neutron?

Obviously, not all these questions can be answered by the first measurements of hyperon form factors we report here, but they indicate the physics potential of such measurements.

We have developed very successful event selection criteria to measure branching fractions for $\psi(2 S) \rightarrow$ hyperons. The figure shows the extremely clean hyperon event distributions as a function of $X=(E(h)+E(\bar{h})) / \sqrt{s}$. 

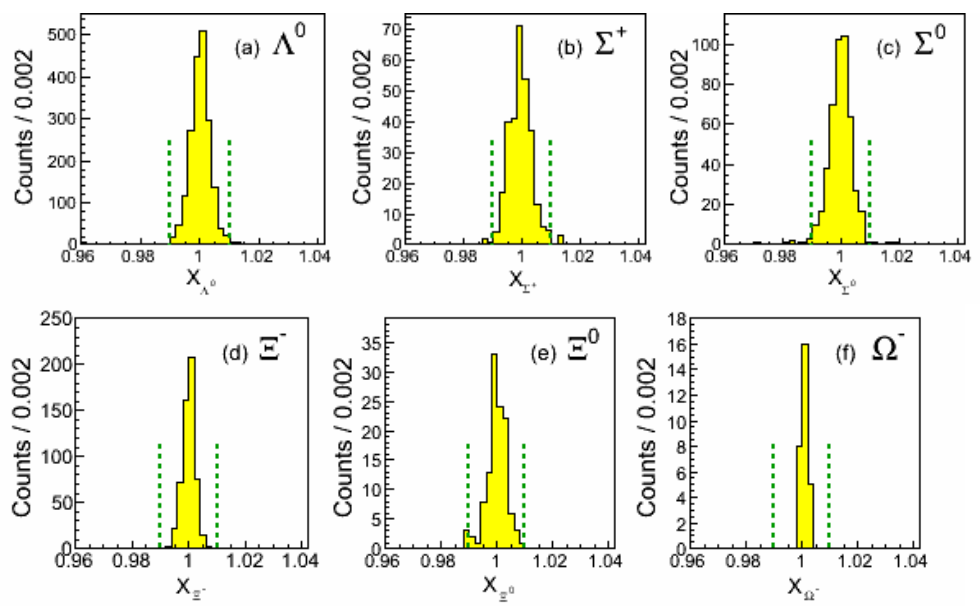

Figure 9. $\mathrm{X}$ distributions for $\psi(2 S) \rightarrow B \bar{B}$

Table 2. The branching factor results for $\psi(2 S) \rightarrow B \bar{B}$.

\begin{tabular}{|c|c|c|c|c|c|c|}
\hline & \multicolumn{2}{|c|}{$\begin{array}{c}\text { CLEO } \\
\text { PRD 72, } 051108 \text { (2005) }\end{array}$} & \multicolumn{3}{|c|}{ Present } \\
\hline & & $N$ & $\begin{array}{c}\mathcal{B} \\
\times 10^{4}\end{array}$ & $\mathrm{~N}$ & $\begin{array}{c}\sigma \\
\mathrm{pb}\end{array}$ & $\begin{array}{c}\mathcal{B} \\
\times 10^{4}\end{array}$ \\
\hline$p$ & $(u u d)$ & $557(24)$ & $2.87(19)$ & $4475(78)$ & $196(3)(12)$ & $3.08(5)(18)$ \\
\hline$\Lambda^{0}$ & (uds) & $208(14)$ & $3.28(34)$ & 1901(44) & $247(6)(15)$ & $3.75(9)(23)$ \\
\hline$\Sigma^{0}$ & (uds) & $58(8)$ & $2.63(41)$ & $439(21)$ & $148(7)(11)$ & $2.25(11)(16)$ \\
\hline$\Sigma^{+}$ & (uus) & $35(6)$ & $2.57(81)$ & 281(17) & $165(10)(11)$ & $2.51(15)(16)$ \\
\hline$\Xi^{-}$ & $(d s s)$ & $63(8)$ & $2.38(37)$ & $548(23)$ & $176(8)(13)$ & $2.66(12)(20)$ \\
\hline$\Xi^{0}$ & (uss) & $19(4)$ & $2.75(88)$ & $112(11)$ & $135(13)(10)$ & $2.02(19)(15)$ \\
\hline$\Omega^{-}$ & (sss) & $4(2)$ & $0.70_{-0.34}^{+0.56}$ & $27(5)$ & $31(6)(3)$ & $0.47(9)(5)$ \\
\hline
\end{tabular}

The branching factor results for $\psi(2 S) \rightarrow B \bar{B}$ are presented in Table 2 . They represent significant improvement over the results reported earlier by CLEO [15].

Using the same event selections as for $\psi(2 S) \rightarrow B \bar{B}$ decays we have analyzed our data of luminosity $=805 \mathrm{pb}^{-1}$ at $\sqrt{s}=3.772 \mathrm{GeV}$, or $\left|\mathrm{Q}^{2}\right|=14.2 \mathrm{GeV}^{2}$ for form factor decays of hyperons. The distributions of the $\mathrm{X}$ parameters are shown in Figure 10.

The number of events $N(B \bar{B})$ in the signal region leads to the Born cross section $\sigma=N(B \bar{B}) /(\epsilon \mathcal{L} C)$, where $\varepsilon$ is the MC-determined efficiency, $\mathcal{L}=802 \mathrm{pb}^{-1}$ is the $e^{+} e^{-}$luminosity, and $\mathrm{C}=0.76-0.78$ is the correction factor for initial state radiation. The cross section is related to the form factors as

$$
\sigma(s)=\left(4 \pi \alpha^{2} \beta_{B} / 3 s\right)\left[\left|G_{M}(s)\right|^{2}+\tau / 2\left|G_{E}(s)\right|^{2}\right]
$$

In Table 3 we quote our results for form factor cross sections and for $G_{M}^{3}\left(14.2 \mathrm{GeV}^{2}\right)$ assuming $G_{E}^{B}(s)=G_{M}^{B}(s)$ for both charged and neutral hyperons, because at $s \equiv\left|Q^{2}\right|=14.2 \mathrm{GeV}^{2}$, finite values of $G_{E}(s)$ are possible even for the neutral hyperons. If $G_{E}^{B}(s)=0$ is assumed $G_{M}^{B}(s)$ results are $\sim 10 \%$ larger.

As anticipated by Cabibo and Gatto, a comparison of Tables 2 and 3 shows that the form factor cross sections for all hyperons are nearly a factor 200 smaller than those for the $\psi(2 S)$ resonance decays. 

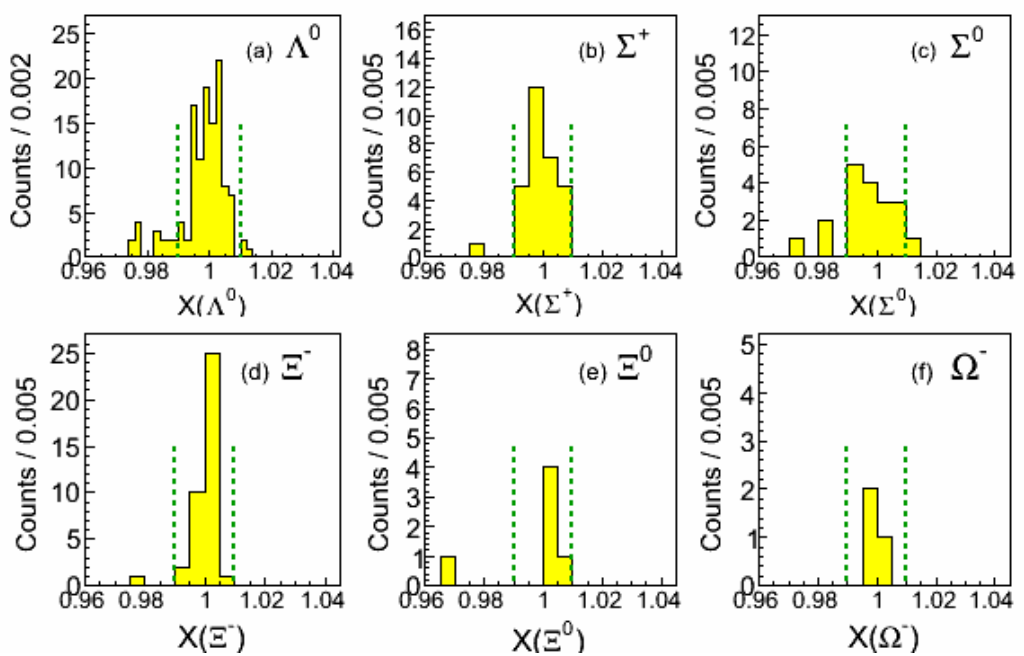

Figure 10. $\mathrm{X}$ distributions for $\psi(3772) \rightarrow B \bar{B}$ for form factor events.

Table 3. The form factor results for the proton and the hyperons. Note that $G_{M}\left(\Lambda^{0}\right)=1.66(23) G_{M}\left(\Sigma^{0}\right)$.

\begin{tabular}{ll|rcc}
\hline \hline & & $N(B \bar{B})$ & $\sigma(B \bar{B})$ & $\begin{array}{c}G_{M}(B \bar{B}) \\
\times 10^{2}\end{array}$ \\
\hline$p$ & $(u u d)$ & $213(15)$ & $0.46(3)(3)$ & $0.88(3)(2)$ \\
$\Lambda^{0}$ & $(u d s)$ & $105(10)$ & $0.80(8)(5)$ & $\mathbf{1 . 1 8}(\mathbf{6})(4)$ \\
$\Sigma^{0}$ & $(u d s)$ & $15(4)$ & $0.29(7)(2)$ & $0.71(9)(3)$ \\
$\Sigma^{+}$ & $(u u s)$ & $29(5)$ & $0.99(18)(6)$ & $1.32(13)(4)$ \\
$\Xi^{-}$ & $($dss $)$ & $38(6)$ & $0.71(11)(5)$ & $1.14(9)(4)$ \\
$\Xi^{0}$ & $(u s s)$ & $5_{-2}^{+3}$ & $0.35_{-0.16}^{+0.20}(3)$ & $0.81(21)(3)$ \\
$\Omega^{-}$ & $(s s s)$ & $3_{-1}^{+2}$ & $0.16_{-0.10}^{+0.13}(2)$ & $0.64_{-0.25}^{+0.21}(3)$ \\
\hline \hline
\end{tabular}

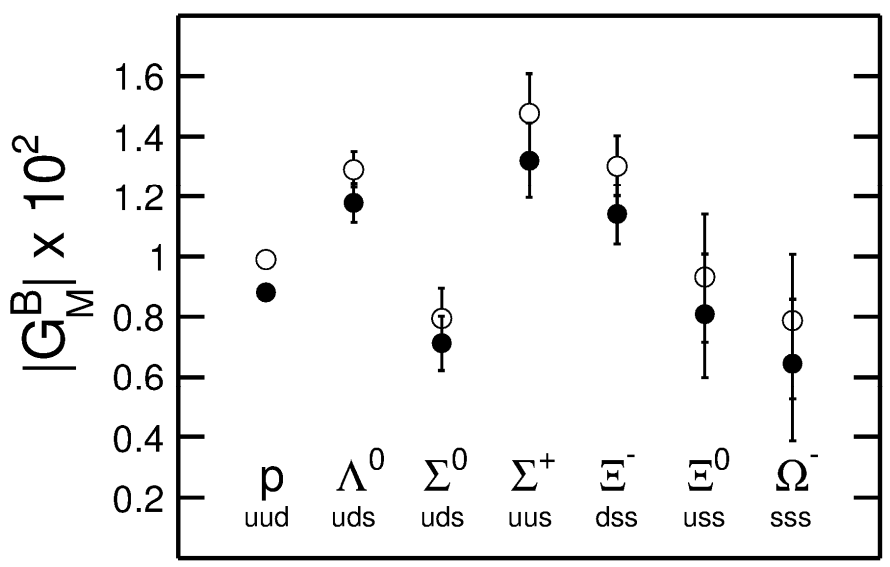

Figure 11. Closed circles: $G_{E}=G_{M}$, Open circles: $G_{E}=0$ 
What do the hyperon form factors tell us : No modern predictions of hyperon form factors for $\mid \mathrm{Q}^{2} \mathrm{I}$ $>1 \mathrm{GeV}^{2}$ exist. The old (1977) VDM-based predictions of $\sigma\left(e^{+} e^{-} \rightarrow B \bar{B}\right)$ by Körner \& Kuroda [16] are factor $\sim 10$ smaller than what we measure. We can therefore only discuss qualitative features and patterns in our data.

1. No evidence is seen for $G_{M}(B)$ being proportional to $\mu_{B}$. Actually, none is expected for timelike form factors.

2. $G_{M}(B)$ vary from $0.6 \times 10^{-2}$ to $1.3 \times 10^{-2}$ relatively smoothly, except for $\Sigma^{0}$.

3. $G_{M}\left(\Lambda^{0}\right)=(1.7 \pm 0.2) \times G_{M}\left(\Sigma^{0}\right)$. Both have $|u d s\rangle$ construct. What does this imply?

4. $G_{M}\left(\Sigma^{+}\right)=1.3 \pm 0.1 \approx G_{M}\left(\Xi^{-}\right)=1.1 \pm 0.1$. Is their near equality due to spin correlations? In both $\Sigma^{+}$(uus) and $\Xi^{-}$(dss) the two like-quarks are coupled to spin-singlet, $\mathrm{S}=0$.

\section{Diquark Correlations}

There is a dramatic difference between $\Lambda^{0}$ and $\Sigma^{0}$ with $G_{M}=(1.7 \pm 0.2) \times G_{M}\left(\Sigma^{0}\right)$. What does it imply?

Both $\Lambda^{0}$ and $\Sigma^{0}$ have a |uds $\rangle$ construct. Why then is $G_{M}\left(\Lambda^{0}\right)$ is $\sim 70 \%$ larger than $G_{M}\left(\Sigma^{0}\right)$. We note that the isospins of $\Lambda^{0}$ and $\Sigma^{0}$ are different: $I\left(\Lambda^{0}\right)=0, I\left(\Sigma^{0}\right)=1$. Since only the $\mathrm{u}$ and $\mathrm{d}$ quarks carry isospin, it is extremely suggestive that the observed difference in $G_{M}$ arises due to differences in the configurations of the $\mathrm{u}$ and d quarks in $\Lambda^{0}$ and $\Sigma^{0}$.

The spins in isoscalar $\Lambda^{0}$ are coupled to $\mathrm{u} \uparrow+d \downarrow$, or $S=0$, and the spins in isovector $\Sigma^{0}$ are coupled to $S=1$. This leads to much stronger spatial correlation between the u and d quarks in $\Lambda^{0}$ compared to $\Sigma^{0}$. With large $\left|\mathrm{Q}^{2}\right|=14.2 \mathrm{GeV}^{2}$ our measurements are sensitive to it. We suggest that this gives rise to $G_{M}\left(\Lambda^{0}\right)$ being much larger than $G_{M}\left(\Sigma^{0}\right)$.

We believe that this explanation based on the diquark correlations between the two light quarks, $\mathrm{u}$ and $\mathrm{d}$ is quite reasonable!

Two-body (fermion) correlations are known to play an important role in many aspects of physics, ranging from Cooper pairs in superconductivity, to pairing interactions in nuclear physics. Diquarkquark models of nucleons have been proposed to explain many observations in hadron physics, particularly the observed $G_{M}$ (timelike) $/ G_{M}$ (spacelike) $\approx 2$ for the proton.

Recently, Wilczek and colleagues [17] have drawn attention to the fact that "it is plausible that several of the most profound aspects of low-energy QCD dynamics are connected to diquark correlations." Wilczek goes on to actually state that

- "The $\Lambda$ is isosinglet, so it features the good diquark [ud], while $\Sigma$, being isotriplet, features the bad diquark (ud)."

- "the good diquark would be significantly more likely to be produced than the bad diquark", and that "this would reflect in a large $\Lambda / \Sigma$ ratio."

We claim that this is exactly what we are observing in the difference between $G_{M}\left(\Lambda^{0}\right)$ and $G_{M}\left(\Sigma^{0}\right)$ and $\sigma\left(\Lambda^{0}\right) / \sigma\left(\Sigma^{0}\right) \approx 3$. Our observations of hyperon form factors thus constitute one of the best evidence for diquark correlations.

\section{References}

1. N. Cabibbo and R. Gatto, Phys. Rev. Lett. 4, 313 (1960).

2. N. Cabibbo and R. Gatto, Phys. Rev. 124, 1577 (1961).

3. S. J. Brodsky and G. R. Farrar, Phys. Rev. Lett. 31, 1153 (1973).

4. T. A. Appelquist and H. D. Politzer, Phys. Rev. Lett. 34, 43 (1975). For a compilation, see Kamal. K. Seth, J. Phys. Conf. Ser. 9, 32 (2005), arXiv: hep-ex / 0501022.

5. T. Horn et al., Phys. Rev. Lett. 97, 192001 (2006), V. Tadavosian et al., Phys. Rev. C 75, 055205 (2007). 
6. K. K. Seth, S. Dobbs, Z. Metreveli, A. Tomaradze, T. Xiao and G. Bonvicini, Phys. Rev. Lett. $110(2), 022002$.

7. S. J. Brodsky and G. P. Lepage, Phy. Rev. D 24, 2824 (1981).

8. A. P. Bakulev, A. V. Radyushkin, and N. G. Stefanis, Phys. Rev. D 62, 113001 (2000). A. P. Bakulev, A. V. Radyushkin, and N. G. Stefanis, Phys. Rev. D 62, 113001 (2000). T. Gousset and B. Pire, Phys. Rev.D 51, 15 (1995). T. Gousset and B. Pire, Phys. Rev.D 51, 15 (1995). G. F. de Teramond and S. J. Brodsky, arXiv: 1203.4025 (private communication).

9. G. P. Lepage and S. J. Brodsky, Phys. Rev. D 22, 2157 (1980).

10. V.M. Braun et al., Phys. Rev. D 74,074501 (2006).

11. K. K. Seth, S. Dobbs, A. Tomaradze, T. Xiao and G. Bonvicini, Phys. Lett. B 730, 332 (2014).

12. T. A. Armstrong et al., Phys. Rev. Lett. 70, 1212 (1993); also, Phys. Rev. D 60, 032002 (1999).

13. P. Kroll et al., Phys. Lett. B 316, 546 (1993).

14. S. Dobbs, A. Tomaradze, T. Xiao, K. K. Seth and G Bonvicini., Phys. Lett. B (in press).

15. T. K. Pedlar et al., (CLEO Collaboration), Phys. Rev.D 72, 051108.

16. J. G. Korner and M. Kuroda, Phys. Rev. D 16, 2165 (1977).

17. R. Jaffe and F. Wilczek, Phys. Rev. Lett. 91, 232003 (2003); also, A. Selem and F. Wilczek, arXiv: hep-ph/ 0602128. 